\title{
Vorinostat enhances chemosensitivity to arsenic trioxide in K562 cell line
}

Nainong Li, Xiaoyan Guan, Fang Li, xiaofan li, Yuanzhong Chen

Objective : This study aimed to investigate the chemosensitive augmentation effect and mechanism of HDAC inhibitor Vorinostat (SAHA) in combination with arsenic trioxide (ATO $)$ on proliferation and apoptosis of K562 cells. Methods = The CCK-8 assay was used to compare proliferation of the cells. Annexin-V and PI staining by flow cytometry and acridine orange/ethidium bromide stains were used to detect and quantify apoptosis. Western blot was used to detect expression of p21, Akt, pAkt, p210, Acetyl-Histone H3, and Acetyl-Histone $\mathrm{H} 4$ proteins. Results = SAHA and ATO inhibited proliferation of K562 cells in an additive and time- and dose-dependent manner. SAHA in combination with ATO showed significant apoptosis of K562 cells in comparison to the single drugs alone ( $p$ $<0.01)$. Both SAHA and ATO alone and in combination showed lower levels of p210 expression. SAHA and SAHA and ATO combined treatment showed increased levels of Acetyl-Histone $\mathrm{H} 3$ and Acetyl-Histone $\mathrm{H} 4$ protein expression. SAHA alone showed increased expression of p21, while ATO alone and in combination with SAHA showed no significant change. SAHA and ATO combined therapy showed lower levels of Akt and pAkt protein expression than SAHA or ATO alone. Conclusion = SAHA and ATO combined treatment inhibited proliferation, induced apoptosis, and showed a chemosensitive augmentation effect on K562 cells. The mechanism might be associated with increasing histone acetylation levels as well as regulating the Akt signaling pathway. 
4 Running title: combination treatment of SAHA and ATO on K562 cell line

5

Word counts: Abstract 236; Text: 2142

16

Key words: histone deacetylase inhibitors; arsenic trioxide; K562 cell; apoptosis. Sponsored by National and Fujian Provincial Key Clinical Specialty Discipline Construction Program, P. R.C 


\section{Abstract:}

32 Objective: This study was aimed to investigate the chemosensitive augmentation effect and mechanism of HDAC 33 inhibitor Vorinostat (SAHA) in combination with arsenic trioxide (ATO) on proliferation and apoptosis of K562 34 cells.

35 Methods: The CCK-8 assay was used to compare proliferation of the cells. Annexin-V and PI staining by flow 36 cytometry and acridine orange/ethidium bromide stains were used to detect and quantify apoptosis. Western blot was 37 used to detect expression of p21, Akt, pAkt, p210, Acetyl-Histone H3, and Acetyl-Histone H4 proteins.

38 Results: SAHA and ATO inhibited proliferation of K562 cells in an additive and time- and dose-dependent manner.

39 SAHA in combination with ATO showed significant apoptosis of K562 cells in comparison to the single drugs alone 40 ( $\mathrm{p}<0.01)$. Both SAHA and ATO alone and in combination showed lower levels of p210 expression. SAHA and 41 SAHA and ATO combined treatment showed increased levels of Acetyl-Histone H3 and Acetyl-Histone H4 protein 42 expression. SAHA alone showed increased expression of $\mathrm{p} 21$, while ATO alone and in combination with SAHA 43 showed no significant change. SAHA and ATO combined therapy showed lower levels of Akt and pAkt protein 44 expression than SAHA or ATO alone.

45 Conclusion: SAHA and ATO combined treatment inhibited proliferation, induced apoptosis, and showed a 46 chemosensitive augmentation effect on K562 cells. The mechanism might be associated with increasing histone 47 acetylation levels as well as regulating the Akt signaling pathway.

48

49

\section{Keywords:}

51 histone deacetylase inhibitors; arsenic trioxide; K562 cell; apoptosis.
52

53

54

55

56

57 


\section{Material and Methods}

\section{Introduction:} (1)

\section{6}

\section{7}

\section{8} 79 80 81

Histone acetylation and deacetylation comprise one of the common modifications found in epigenetics.

Histone acetylation helps transcription factors bind to DNA templates and activates transcription;

however, histone deacetylation inhibits transcription. ${ }^{[1-4]}$ In 2006, the second generation of hydroxamic acids histone deacetylase inhibitor (HDACi) SAHA (suberoylanilide hydroxamic acid, Vorinostat) was approved by the FDA for clinical treatment of relapsed or refractory cutaneous T-cell lymphoma. [5] Chronic myeloid leukemia $(\mathrm{CML})$ is a malignant clonal disease derived from hematopoietic stem cells, and it manifests itself through characteristic molecular markers: the bcr-abl fusion gene and the expression of p210 bcr-abl fusion protein. Tyrosine kinase inhibitors (TKI) are the first line of treatment for CML. Although TKIs target the bcr-abl gene, they are unable to eradicate the malignant clone completely. Moreover, drug resistance has been observed in clinical applications. To better eradicate the $\mathrm{CML}$ clone and overcome the drug resistance, we hypothesized that SAHA augments chemosensitivity when combined with Arsenic trioxide (ATO), another widely used chemoreagent for clinical treatment of hematologic malignancies. Thereby, we investigated the anti-leukemia effect of SAHA combined with ATO on the K562 cell line. 
84 1. Reagent and cell lines

85 SAHA was kindly provided by Dr. Defu Zeng (City of Hope National Medical Center, US, 91010).

86 DMSO was diluted to a concentration of $10 \mathrm{mmol} / \mathrm{L}$. ATO was purchased from Heilongjiang Harbin

87 Medical University Pharmaceutical Co., Ltd., and diluted to a concentration of $2 \mathrm{mmol} / \mathrm{L}$. Both SAHA 88 and ATO were stored at $-20^{\circ} \mathrm{C}$. RPMI 1640 was purchased from Gibco; fetal bovine serum was 89 purchased from TBD; penicillin / streptomycin was purchased from Hyclone; an apoptosis detection kit 90 (Annexin-V-FITC, PI double staining) was purchased from Roche; Protein Extraction reagents were 91 purchased from Wuhan Boster Biological Co., Ltd.; antibodies against c-abl, p21, Acetyl-Histone H3 92 and Acetyl-Histone H4, and other proteins were purchased from Cell Signaling Technology. The K562 93 cell line was preserved and cultured in Fujian Institute of Hematology with a standard protocol as 94 previously described. [6,7]

95

96

97

98

99

100

101

102

103

104

105

106

107

108

109

\section{Proliferation assay}

A CCK-8 assay was used to detect cell line proliferation. A positive control, negative control and the drug treated group (SAHA: 0.25,0.5,1,2 $\mu \mathrm{mol} / \mathrm{L}$; ATO: 1,2,4,8 $\mu \mathrm{mol} / \mathrm{L}$ ) were detected. The assay was completed in triplicate for each group. Next, $5 \times 10^{4}$ cells $/ \mathrm{ml}$ were seeded into a 96-well culture plate to a final volume of $100 \mu \mathrm{l}$. After 24,48 , and $72 \mathrm{~h}$ of treatment, $10 \mu \mathrm{l}$ of CCK-8 was added into each well and incubated for $1-4 \mathrm{~h}$ at $37^{\circ} \mathrm{C}$ in a $5 \% \mathrm{CO}_{2}$ incubator. The absorbance at $450 \mathrm{~nm}$ was measured using a microplate reader. The inhibition rate was calculated using the following equation: Inhibition rate $=[$ (control group - experimental group) $/$ blank group $] \times 100 \%$. A proliferation curve was plotted based on the drug concentration and the proliferation inhibition rate. The $Q$ value was calculated to measure the combined effect of SAHA and ATO. Q was calculated using the following equation: $\mathrm{Q}=\mathrm{E}(\mathrm{ab}) /(\mathrm{Ea}+\mathrm{Eb}-\mathrm{Ea} \times \mathrm{Eb})$ as previously described. ${ }^{[6,7]}$ Generally, Ea and Eb are the single drug inhibition rates; $E(a b)$ is associated with the inhibition rate of SAHA and ATO. Q > 1.15 was considered to be a synergistic effect, $0.85>Q>1.15$ an additive effect, and $Q<0.85$ an antagonistic effect. 
111 3. Annexin-V and PI staining

112 Apoptosis was detected by Annexin-V and PI staining. Generally, K562 cells $\left(1 \times 10^{6}\right)$ and the 113 appropriate drugs were co-incubated for $48 \mathrm{~h}$. Cells were harvested after a single wash with PBS.

114 Next, binding buffer $(100 \mu \mathrm{l})$ was added to resuspending cells. Annexin-V $(2 \mu \mathrm{l})$ and PI $(2 \mu \mathrm{l})$ were

115 added, and the cells were incubated at room temperature for 10-15 min in the dark. K562 cell were 116 detected by flow cytometry. Annexin- $\mathrm{V}^{-/} \mathrm{Pl}-$ cells were living cells, and Annexin- $\mathrm{V}^{+} / \mathrm{Pl}^{-}$cells were early 117 apoptotic cells. Annexin-V + / PI + were late apoptotic cells. This experiment was repeated three times.

119 4. AO / EB fluorescence staining

120 K562 cells and the drugs were co-incubated as described above. Suspended cells (95 $\mu$ l, $\left.5 \times 10^{6} / \mathrm{ml}\right)$

121 were mixed with AO / EB (5 $\mu \mathrm{l})$. Immediately after mixing, one drop of suspended cells was placed on

122 a clean glass slide and observed by fluorescence microscopy at an excitation wavelength of $490 \mathrm{~nm}$.

123 Cells with green fluorescence in the nucleus and cytoplasm were normal cells; cells with yellow-green

124 fluorescence in the nucleus or cytoplasm were apoptotic cells; cells with red fluorescence in nucleus 125 were necrotic cells.

127 5. Western Blot

128 K562 cells were co-cultured as described above. After the cells were lysed, the supernatant was 129 collected and quantified. Total protein $(40 \mu \mathrm{g})$ was transferred to a PVDF membrane after SDS-PAGE 130 electrophoresis. After blocking at room temperature for $2 \mathrm{~h}$, the primary antibodies (against p210, p21, 131 Acetyl-Histone $\mathrm{H} 3$, Acetyl-Histone $\mathrm{H} 4$,Akt and pAkt) were added and incubated at $4{ }^{\circ} \mathrm{C}$ overnight. 132 After washing, the secondary antibody (horseradish peroxidase-conjugated anti-mouse lgG) was 133 added and incubated for $2 \mathrm{~h}$. The chemiluminescence reaction was performed. $\beta$-actin was used as 134 an internal control.

135

136 6. Statistical analysis 
137 Statistical analysis was performed with SPSS 11.5. Means were compared by the Dunnett-t test, with

$138 \mathrm{P}<0.05$ considered to be a significant difference.

140 Results

SAHA in combination with ATO showed an additive effect on the inhibition of proliferation

Compared with the control group, SAHA and ATO inhibited proliferation of K562 cells in a time- and dose-dependent manner. After the cells were treated with SAHA and ATO, cell proliferation was significantly inhibited (Fig. 1). SAHA in combination with ATO showed an additive effect on the inhibition of proliferation (Table 1).

SAHA in combination with ATO showed more apoptosis than the single drugs alone

Annexin-V-FITC/PI double staining flow cytometry can distinguish early apoptotic cells, late apoptotic cells and necrotic cells. In the lower left quadrant are normal cells; in the lower right quadrant are early apoptotic cells; in the upper right quadrant are late apoptotic cells; in the upper-left quadrant are necrotic cells. The results showed that $95 \%$ of the cells in the control group were living cells; the single drug apoptosis rates increased with increasing drug concentration. The K562 cells apoptosis rates were $10.85 \pm 0.65 \%, 29.65 \pm 1.75 \%$, and $84.00 \pm 0.80 \%, 48 \mathrm{~h}$ after treatment with SAHA $(2 \mu \mathrm{mol} / \mathrm{L})$, ATO $(8 \mu \mathrm{mol} / \mathrm{L})$ and $8 \mu \mathrm{mol} / \mathrm{L}$ ATO combined with $2 \mu \mathrm{mol} / \mathrm{L}$ SAHA, respectively. SAHA in combination with ATO showed significant apoptosis of K562 cells compared to the single drugs alone ( $p<0.01$, Fig. 2A).

161 The apoptosis results were confirmed by AO/EB staining. In the control group, K562 cells showed 162 uniform cell size and morphology and homogeneous green fluorescence in the nucleus and cytoplasm. 
163 In contrast, $48 \mathrm{~h}$ after SAHA and ATO combined treatment, we found differences in cell size and

164 morphology. The nucleus was dense, showing yellow-green fluorescent debris. Additionally, SAHA in 165 combination with ATO showed more apoptosis characteristics than the single drugs alone (Fig. 2B-E).

166

167

168

169

170

171

172

173 174 (Fig. 3).

175

176

177

178

179

180

181

182

183

184

185

186

187

188 CTCL.

\section{SAHA in combination with ATO showed pronounced changes in protein expression}

Both SAHA and ATO alone and in the combination group showed lower levels of p210 expression and. The SAHA group and the combination group showed increased levels of Acetyl-Histone H3 and Acetyl-Histone H4 protein expression. SAHA alone showed increased levels of p21 WAF1/CIP1 expression, while ATO alone and in the combination group showed no significant changes. The combination group showed lower levels of Akt and pAkt protein expression than SAHA or ATO alone

\section{Discussion}

There are a variety of HDACls undergoing clinical trials for the treatment of solid tumors and hematological malignancies, especially for the treatment of cutaneous T-cell lymphoma (CTCL), peripheral T-cell lymphoma, and Hodgkin's lymphoma, which have shown promising results. ${ }^{[8]}$ As a novel HDACI, SAHA was approved by the FDA for the treatment of progressive clinical recurrence of

ATO is an important medicine for treating leukemia (particularly for acute promyelocytic leukemia, APL). Additionally, chemotherapy regimens based on ATO are a popular research topic. In recent years, the mechanism and clinical applications of ATO have been reported, with induction of oxidative stress and DNA damage included. ${ }^{[9,10]}$ Our study mainly focused on the Akt signal pathway. Huang 
189

190

191

192

193

194

195

196

197

198

199

200

201

202

203

204

205

206

207

208

209

210

211

212

213

213 Akt is a serine / threonine protein kinase, which plays a major role in the anti-apoptotic pathway in
215 apoptosis signaling. ${ }^{[12]}$ We found that both the SAHA group and ATO group downregulate Akt and

213 Akt is a serine / threonine protein kinase, which plays a major role in the anti-apoptotic pathway in
215 apoptosis signaling. ${ }^{[12]}$ We found that both the SAHA group and ATO group downregulate Akt and

reported that ATRA and arsenic compound-based combination therapy was effective in re-inducing morphological remission in relapsed patients with APL who had previous exposure to ATRA and arsenic compounds, thus producing low molecular remission rates and high risk of secondary relapse. [11] Interestingly, we also found chemosensitive augmentation of SAHA in the NB4 cell line (data not shown), which indicates that combined SAHA and ATO therapy could have clinical applications in the treatment of leukemia.

The CCK8 results show that individual treatments with SAHA or ATO inhibited K562 cell proliferation in a time- and dose-dependent manner. The combination treatment showed an additive effect in inhibiting proliferation. Additionally, experimental results showed that at certain combined concentrations of SAHA and ATO, there was a greater level of apoptosis than the single drugs alone, which induced apoptosis over $80 \%$. These results indicated that the combined treatment might have beneficial effects including improved chemotherapeutic effects and minimized side effects.

In this study, both SAHA and the combination group induced increased expression of Acetyl-Histone $\mathrm{H} 3$ and Acetyl-Histone H4 when compared to ATO and the control. This outcome indicates that SAHA can significantly increase histone acetylation levels and activate transcription, thereby inducing hematological tumor cell apoptosis. ${ }^{[3,4]}$

p21 is an important cell cycle regulatory protein involved in cell growth, differentiation, aging and death. p21 plays a negative role in the regulation of the cell cycle by acting on CDKI. p21 expression was upregulated after treatment with SAHA compared to ATO and the control, thereby blocking the cell cycle and inducing apoptosis. Interestingly, combination therapy did not induce expression of p21. This mechanism needs to be studied further. 
216 pAkt expression. Compared to the single drug treatment, the combination group downregulated Akt 217 and pAkt expression drastically, indicating that the mechanism may be associated with the regulation 218 of the Akt signaling pathway.

220 p210 fusion protein is the primary cause of CML pathogenesis and progression. Furthermore, the 221 p210 fusion protein is the source of the abnormal phenotype of chronic phase CML. We found that 222 both the SAHA group and the ATO group downregulate p210 fusion protein expression. Also the 223 combination group showed downregulation of p210 protein. Given the dosage which we applied by 224 proliferation experiments, both SAHA and ATO in the concentration are very effective in 225 downregulating the p210 fusion protein expression. It could be more effective by the combination.

226 Taken together, these results indicated that both SAHA and ATO can induce cell apoptosis and 227 inhibition of cell proliferation by downregulating the characteristic p210 fusion protein in K562 cells.

228 Combining SAHA with ATO also lowered the expression of $\mathrm{p} 210$, which resulted in increased 229 apoptosis.

231 In summary, SAHA and ATO can significantly inhibit the proliferation of K562 cells and induce apoptosis. 232 Combined treatment can enhance apoptosis effects within a certain concentration range. The 233 mechanism may be associated with increased histone acetylation and the regulation of the Akt 234 signaling pathway. This study may provide a new combination chemotherapy regimen for the treatment 235 of CML. Further in vivo experiments and animal model should be set up to test the enhanced 236 chemosensitivity effect by combining SAHA and ATO. We also need to observe the side effects 237 by combining SAHA and ATO and prepare data for translational medicine work.

\section{References:}

[1]Ciro M, Saverio M, Pier G P. Histone deacetylases and epigenetic therapies of hematological malignancies. Pharmacol Res. 2010;62: $18-34$.

[2] Wang GG, Allis CD, Chi P. Chromatin remodeling and cancer, Part I:Covalent histone modifications. Trends Mol Med. 2007; 13:363 - 372.

[3]Reid G, Gallais R. Marking time: the dynamic role of chromatin and covalent modification in 
transcription. Int J Biochem Cel1 Biol. 2009; 41:155- 163.

[4]Victoria M. R, Jose G, James S. H. Development of vorinostat Current applications and future perspectives for cancer therapy. Cancer Lett. 2009;280:201-210.

[5]Siegel D, Hussein M, Belani C, Robert F, Galanis E, Richon V, et al. Vorinostat in solid and hematologic malignancies. J Hematol 0ncol. 2009; 2:31.

[6] Liu A, Sun Z, Wang K, Chen X, Xu X, Wu Y, et al. Molecular beacon-based fluorescence biosensor for the detection of gene fragment and PCR amplification products related to chronic myelogenous leukemia. Anal Bioanal Chem. 2012; 402:805-12

[7] Lin L, Chen J, Lin Q, Chen W, Chen J, Yao H, et al. Electrochemical biosensor based on nanogoldmodified poly-eriochrome black $\mathrm{T}$ film for BCR/ABL fusion gene assay by using hairpin LNA probe. Talanta. $2010 ; 80: 2113-9$.

[8] Duvic M, Talpur R, Ni X, Zhang C, Hazarika P, Kelly C, et al. Phase 2 trial of oral vorinostat (suberoylanilide hydroxamic acid, SAHA) for refractory cutaneous T-cell lymphoma (CTCL). Blood. 2007; 109: $31-39$.

[9]Sanjay Kumar, Clement G Yedjou and Paul B Tchounwou. Arsenic trioxide induces oxidative stress, DNA damage, and mitochondrial pathway of apoptosis in human leukemia (HL-60) cells. Journal of Experimental \& Clinical Cancer Research 2014, 33:42

[10] Wilson H. Miller, Jr., Hyman M. Schipper, Janet S. Lee, Jack Singer, and Samuel Waxman. Mechanisms of Action of Arsenic Trioxide. CANCER RESEARCH. 2002: 62, 3893 - 3903,

[11] Lu J, Huang X, Bao L, Jiang H, Zhu H, Jiang B. Treatment outcomes in relapsed acute promyelocytic leukemia patients initially treated with all-transretinoic acid and arsenic compound-based combined therapies. Oncol Lett. 2014;7:177-182.

[12] Osaki M, Oshimura M, Ito H. PI3K-Akt pathway: its functions and alterations in human cancer. Apoptosis. 2004; 9: 667-676.

\section{Conflict of interest: The authors declare no conflict of interests.}




\section{Table $\mathbf{1}_{\text {(on next page) }}$}

Tab.1: SAHA in combination with ATO showed an additive effect on the inhibition of proliferation.

$5 \times 10^{4}$ cells / $\mathrm{ml}$ were seeded to a 96-well culture plate in a final volume of $100 \mu l$. Different concentration of drugs were applied. Inhibition rate was calculated as Fig.1. Q was calculated using the following equation: $\mathrm{Q}=\mathrm{E}(\mathrm{ab}) /(\mathrm{Ea}+\mathrm{Eb}-\mathrm{Ea} \times \mathrm{Eb})$. Ea and Eb are the single drug inhibition rates; $E(a b)$ is associated with the inhibition rate of SAHA and ATO. Q > 1.15 was considered to be a synergistic effect, $0.85>\mathrm{Q}>1.15$ an additive effect, and $\mathrm{Q}<0.85$ an antagonistic effect. 
2 Tab.1: SAHA in combination with ATO showed an additive effect on the inhibition of proliferation.

\begin{tabular}{ccccc}
\hline \multicolumn{2}{c}{ Concentration $(\mu$ mol/L) } & Inhibition $(\%)$ & $Q$ value & additive effect \\
\cline { 1 - 2 } SAHA & AT0 & & & \\
\hline 0 & 0 & 0 & & \\
0.25 & 0 & 45.33 & & \\
0.5 & 0 & 62.24 & & \\
1 & 0 & 76.01 & & \\
2 & 0 & 95.84 & & \\
0 & 1 & 3.7 & & \\
0 & 2 & 14.07 & & + \\
0 & 4 & 76.67 & & + \\
0 & 8 & 96.99 & & + \\
0.25 & 1 & 49.91 & 0.906 & + \\
0.25 & 2 & 55.75 & 1.051 & + \\
0.25 & 4 & 84.08 & 0.964 & + \\
0.5 & 1 & 63.85 & 1.003 & + \\
0.5 & 2 & 70.88 & 1.049 & + \\
0.5 & 4 & 85.41 & 0.937 & + \\
1 & 1 & 84.75 & 1.102 & + \\
1 & 2 & 87.19 & 1.098 & + \\
1 & 4 & 96.01 & 1.017 & \\
\hline
\end{tabular}




\section{1}

Fig. 1: SAHA and ATO alone showed inhibition of proliferation on K562 cell line.

$5 \times 10^{4}$ cells / $\mathrm{ml}$ were seeded to a 96 -well culture plate in a final volume of $100 \mu l$. After 24 , 48 , and $72 \mathrm{~h}$ of treatment, $10 \mu \mathrm{l}$ of CCK-8 was added into each well and incubated for 1-4 $\mathrm{h}$. The absorbance at $450 \mathrm{~nm}$ was measured using a microplate reader. Inhibition rate $=$ [(control group - experimental group) / blank group] $\times 100 \%$. (A) The proliferation curve of SAHA alone is shown. (B) The proliferation curve of ATO alone is shown. 


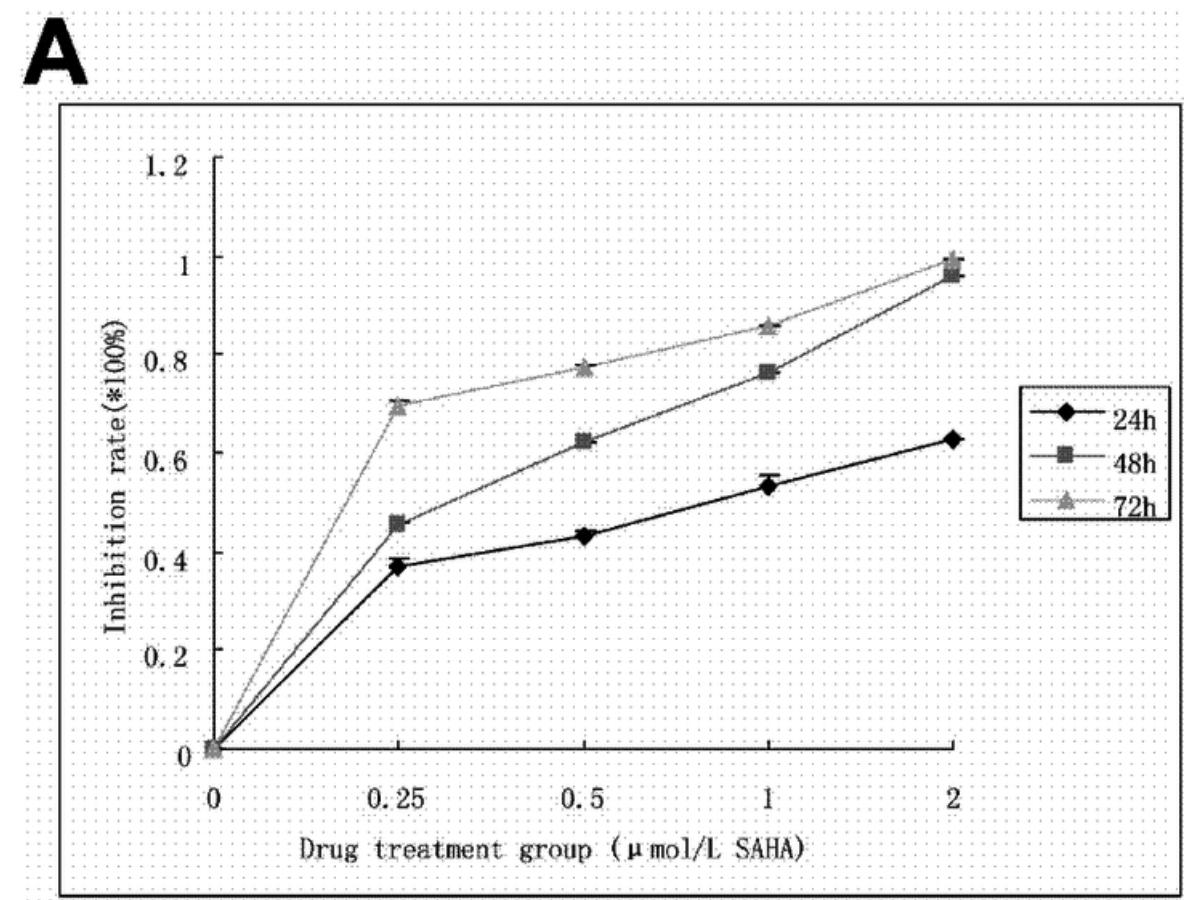

B

\section{SAHA group}

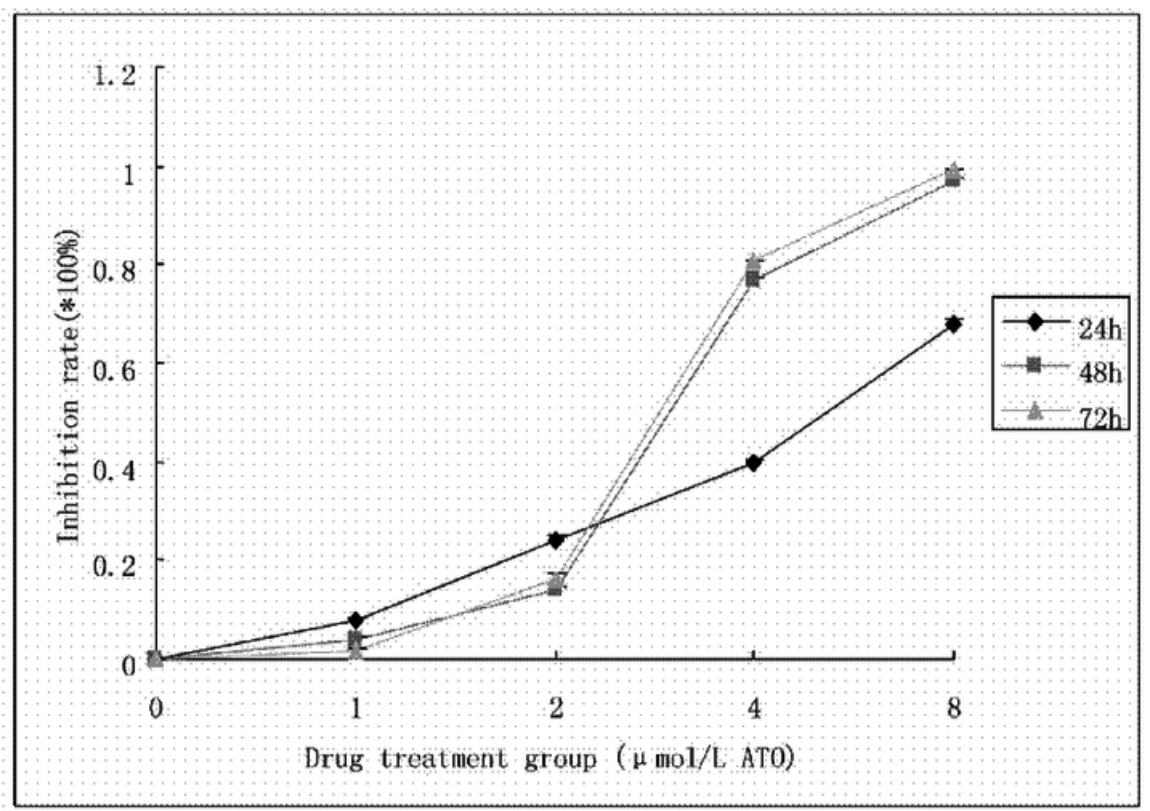

ATO group 


\section{2}

Apoptosis rate after treatment.

(A) Apoptosis was detected by Annexin-V and PI staining. K562 cells $\left(1 \times 10^{6}\right)$ and the drugs

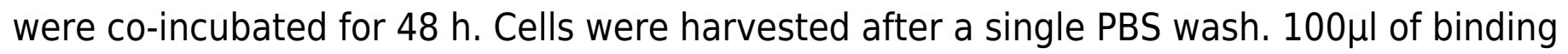
buffer was added to resuspend the cells. Annexin-V $(2 \mu \mathrm{l})$ and PI $(2 \mu \mathrm{l})$ was added. After an incubation at room temperature for 10-15 min in the dark, K562 cells were detected using a Flow Machine. The apoptosis rates after different treatments are shown (SA2:2 $\mu \mathrm{mol} / \mathrm{L}$

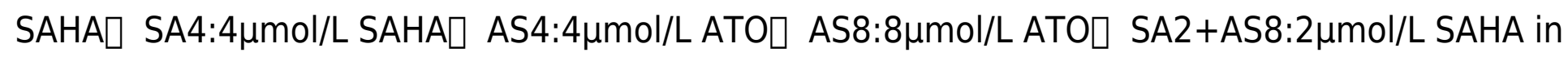
combination with $8 \mu \mathrm{mol} / \mathrm{L}$ ATO).(B-E) Apoptosis observations using fluorescence microscopy $48 \mathrm{~h}$ after treatment $\times 400$ magnification). K562 cells and the drugs were co-incubated as described in Fig. 2. Suspended cells $\left(95 \mu \mathrm{l}, 5 \times 10^{6} / \mathrm{ml}\right)$ were mixed with AO / EB $(5 \mu \mathrm{l})$. Immediately after mixing, one drop of suspended cells was placed on a clean glass slide and observed by fluorescence microscopy at an excitation wavelength of $490 \mathrm{~nm}$. Cells with green fluorescence in the nucleus and cytoplasm were normal cells; Cells with yellow-green fluorescence in the nucleus or cytoplasm were apoptotic cells; cells with red fluorescence in the nucleus were necrotic cells. (B) control $\square$ (C) $2 \mu \mathrm{mol} /$ L SAHA; (D) $8 \mu \mathrm{mol} /$ L ATO (E) 2 $\mu \mathrm{mol} / \mathrm{L}$ SAHA in combination with $8 \mu \mathrm{mol} / \mathrm{L}$ ATO. 


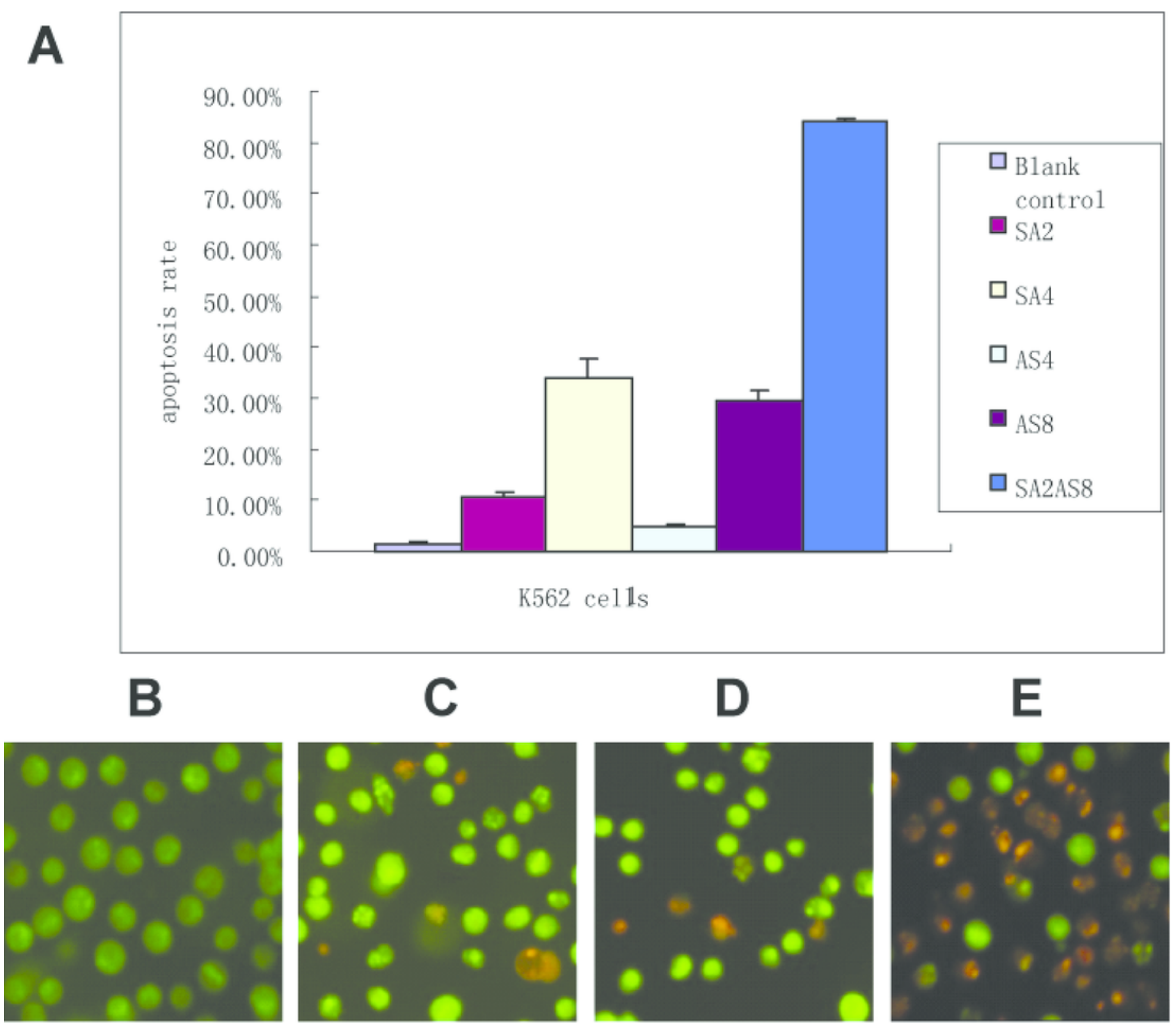


Figure 3 (on next page)

Fig. 3: SAHA in combination with ATO showed pronounced changes in protein expression.

K562 cells were co-cultured as described above. p210, p21, Acetyl-Histone H3, AcetylHistone $\mathrm{H} 4$, Akt and pAkt were detected by Western blot. $\beta$-actin was used as an internal control. (C. control $\square$ S. $2 \mu \mathrm{mol} /$ L SAHA $\square$ A. $8 \mu \mathrm{mol} /$ L ATO $\square$ S+A. $2 \mu \mathrm{mol} / \mathrm{L}$ SAHA in combination with $8 \mu \mathrm{mol} /$ L ATO) 


\section{PeerJ Reviewing Manuscript}

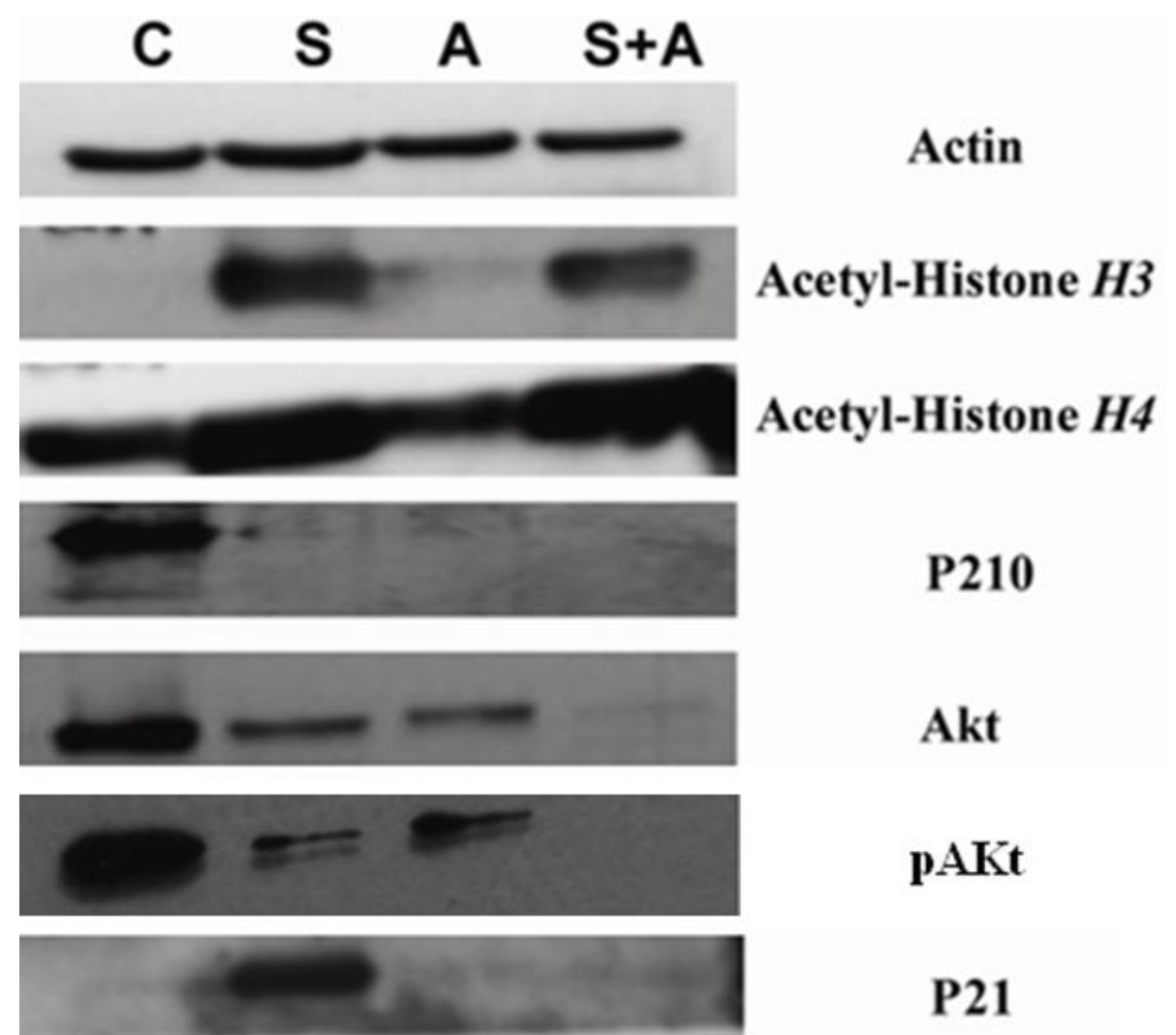

